\title{
EXTREME STAR FORMATION IN THE HOST GALAXIES OF THE FASTEST GROWING SUPERMASSIVE BLACK HOLES AT $z=4.8$
}

\author{
Rivay Mor ${ }^{1}$, Hagai Netzer ${ }^{1}$, Benny Trakhtenbrot $^{1}$, Ohad Shemmer $^{2}$, and Paulina LiRA $^{3}$ \\ ${ }^{1}$ School of Physics and Astronomy and the Wise Observatory, The Raymond and Beverly Sackler Faculty of Exact Sciences, Tel-Aviv University, Tel-Aviv 69978, \\ Israel; rivay@wise.tau.ac.il \\ 2 Department of Physics, University of North Texas, Denton, TX 76203, USA \\ ${ }^{3}$ Departamento de Astronomia, Universidad de Chile, Camino del Observatorio 1515, Santiago, Chile \\ Received 2012 January 12; accepted 2012 March 5; published 2012 March 30
}

\begin{abstract}
We report new Herschel observations of $25 z \simeq 4.8$ extremely luminous optically selected active galactic nuclei (AGNs). Five of the sources have extremely large star-forming (SF) luminosities, $L_{\mathrm{SF}}$, corresponding to SF rates (SFRs) of 2800-5600 $M_{\odot} \mathrm{yr}^{-1}$ assuming a Salpeter initial mass function. The remaining sources have only upper limits on their SFRs, but stacking their Herschel images results in a mean SFR of $700 \pm 150 M_{\odot} \mathrm{yr}^{-1}$. The higher SFRs in our sample are comparable to the highest observed values so far at any redshift. Our sample does not contain obscured AGNs, which enables us to investigate several evolutionary scenarios connecting supermassive black holes and SF activity in the early universe. The most probable scenario is that we are witnessing the peak of SF activity in some sources and the beginning of the post-starburst decline in others. We suggest that all 25 sources, which are at their peak AGN activity, are in large mergers. AGN feedback may be responsible for diminishing the SF activity in 20 of them, but is not operating efficiently in 5 others.
\end{abstract}

Key words: galaxies: active - galaxies: star formation - quasars: general

Online-only material: color figures

\section{INTRODUCTION}

The growth and evolution of supermassive black holes (SMBHs) are closely connected to the formation and evolution of their host galaxies. While SMBHs grow through accretion of matter from their surroundings during an active galactic nucleus (AGN) phase, their host galaxies grow by star formation (SF). The bolometric luminosity of the AGN $\left(L_{\mathrm{AGN}}\right)$ is found to be related to the SF luminosity $\left(L_{\mathrm{SF}}\right.$; defined as the integrated luminosity due to SF between 8 and $1000 \mu \mathrm{m}$ ) in its host galaxy. In AGN-dominated sources (those with $L_{\mathrm{AGN}}>L_{\mathrm{SF}}$ ), there is evidence for a simple power-law relationship between the two luminosities that can be expressed as $L_{\mathrm{SF}} \simeq 10^{43}\left(L_{\mathrm{AGN}} /\left(10^{43} \mathrm{erg} \mathrm{s}^{-1}\right)\right)^{0.7}$ (Netzer 2009). This relationship is found in type-I (Netzer et al. 2007b; Lutz et al. 2008) and type-II (Netzer 2009) sources.

Recent studies provide more insight into the general $L_{\mathrm{AGN}}-L_{\mathrm{SF}}$ dependence. Shao et al. (2010) and Hatziminaoglou et al. (2010) measured $L_{\mathrm{SF}}$ for the hosts of known AGNs in the GOODS-N and HerMES fields, respectively. Both studies find indications that, in SF-dominated sources (i.e., $L_{\mathrm{SF}} \gtrsim L_{\mathrm{AGN}}$ ), there is no clear correlation between the two luminosities. Furthermore, Shao et al. (2010) found that for SF-dominated sources, in a given redshift bin, $L_{\mathrm{SF}}$ is roughly constant with increasing $L_{\mathrm{AGN}}$. This behavior can be interpreted as an indication for different stages of evolution, where in sources with $L_{\mathrm{SF}}>L_{\mathrm{AGN}}$ the SMBH has not yet reached its highest accretion rate phase. These Herschel ${ }^{4}$-based studies included mostly low $L_{\mathrm{AGN}}$ sources and very few sources at $z \geqslant 2.5$. At higher redshifts and higher $L_{\mathrm{AGN}}$ there are very few known SF-dominated sources, although a handful of sources with $L_{\mathrm{SF}} \simeq L_{\mathrm{AGN}}$ have been found mainly by submillimeter (sub-mm) observations

\footnotetext{
4 Herschel is an ESA space observatory with science instruments provided by European-led Principal Investigator consortia and with important participation from NASA.
}

(e.g., Isaak et al. 2002; Priddey et al. 2003; Wu et al. 2009; Leipski et al. 2010).

Galaxy evolution scenarios suggest two modes of SF (see, e.g., Rodighiero et al. 2011). The steadier process of secular evolution is common in isolated disk galaxies and can reach SF rates (SFRs) of $\sim 400 M_{\odot} \mathrm{yr}^{-1}$ at high redshift. A less common process with SFR that can exceed $\sim 1000 M_{\odot} \mathrm{yr}^{-1}$ is associated with mergers between two large gas-rich galaxies (Di Matteo et al. 2005; Guyon et al. 2006; Sijacki et al. 2011; Valiante et al. 2011). Both processes result in cold gas inflow into the center of the system which can trigger AGN activity. Numerical simulations and semi-analytic models of mergers suggest that in such events, the fastest SMBH growth phase succeeds the peak of SF activity by several hundred Myr and is likely to take place when the SMBH is obscured (e.g., Hopkins et al. 2006; Di Matteo et al. 2008). If correct, it means that the most luminous SF phase of the merger precedes the most luminous AGN phase. Observationally, this is supported by the fact that sub-mm galaxies (SMGs) have high $L_{\mathrm{SF}}$, however, they often exhibit little or no AGN activity.

SF and AGN activity may also be related through AGN feedback. This process can diminish or even terminate SF and SMBH accretion through fast winds and intense AGN radiation (e.g., Di Matteo et al. 2005; Springel et al. 2005; Sijacki et al. 2007). The power of AGN feedback depends on $L_{\mathrm{AGN}}$ and can be important in the final stages of both SF modes.

In this Letter we report new Herschel observations of 25 optically selected AGNs from our $z \simeq 4.8$, flux-limited sample (Trakhtenbrot et al. 2011, hereafter T11). Our results provide evidence for extreme SFRs in $20 \%$ of the sources, indicating merger-driven SF activity. In Section 2, we describe the sample and the observations and explain our method for deriving $L_{\mathrm{SF}}$ and SFR. Section 3 compares our findings with earlier results and discusses the way they improve the understanding of the relations between AGN activity and SF in the early universe. 
Table 1

$L_{\mathrm{SF}}$ and SFRs for the Five Individually Detected and Stacked Sources

\begin{tabular}{lccccccc}
\hline \hline Object Name & Redshift & $\begin{array}{c}L_{\mathrm{AGN}}^{\mathrm{a}} \\
\left(10^{47} \mathrm{erg} \mathrm{s}^{-1}\right)\end{array}$ & $\begin{array}{c}f_{250} \\
(\mathrm{mJy})\end{array}$ & $\begin{array}{c}f_{350} \\
(\mathrm{mJy})\end{array}$ & $\begin{array}{c}f_{500} \\
(\mathrm{mJy})\end{array}$ & $\begin{array}{c}L_{\mathrm{SF}^{\mathrm{b}}} \\
\left(10^{13} L_{\odot}\right)\end{array}$ & $\begin{array}{c}\mathrm{SFR}^{\mathrm{c}} \\
\left(M_{\odot} \mathrm{yr}^{-1}\right)\end{array}$ \\
\hline $\mathrm{J} 0331-0741$ & 4.73 & 1.23 & $27.6 \pm 6.2$ & $29.1 \pm 6.7$ & $19.1 \pm 7.0$ & $2.18_{-0.27}^{+0.38}$ & $3771_{-472}^{+653}$ \\
$\mathrm{~J} 0807+1328$ & 4.88 & 1.17 & $13.5 \pm 5.9$ & $22.7 \pm 6.5$ & $21.3 \pm 7.0$ & $1.65_{-0.25}^{+0.45}$ & $2860_{-431}^{+777}$ \\
$\mathrm{~J} 1341+0141$ & 4.69 & 1.82 & $34.5 \pm 6.4$ & $44.0 \pm 7.1$ & $37.0 \pm 7.4$ & $3.26_{-0.28}^{+0.39}$ & $5645_{-479}^{+667}$ \\
$\mathrm{~J} 1619+1238$ & 4.81 & 0.95 & $39.4 \pm 6.5$ & $32.9 \pm 6.8$ & $21.2 \pm 7.0$ & $3.00_{-0.44}^{+0.20}$ & $5184_{-759}^{+346}$ \\
$\mathrm{~J} 2225-0014$ & 4.89 & 1.70 & $23.3 \pm 6.1$ & $26.2 \pm 6.6$ & $18.6 \pm 6.9$ & $2.18_{-0.39}^{+0.29}$ & $3771_{-669}^{+495}$ \\
\hline Stacked source & 4.75 & 0.78 & $4.53_{-1.02}^{+1.24}$ & $6.22_{-1.75}^{+1.62}$ & $3.65_{-1.31}^{+1.43}$ & $0.32_{-0.25}^{+0.25}$ & $697_{-312}^{+359}$ \\
\hline
\end{tabular}

Notes.

a Values taken from T11 except J1619+1238 for which $L_{\mathrm{AGN}}$ was calculated as described in T11.

b Assuming that there is no confusing source within 10" from the AGN.

${ }^{c}$ Assuming a Salpeter initial mass function (IMF).

Throughout this Letter, we assume $H_{0}=70 \mathrm{~km} \mathrm{~s}^{-1} \mathrm{Mpc}^{-1}$, $\Omega_{\mathrm{M}}=0.3$, and $\Omega_{\Lambda}=0.7$.

\section{OBSERVATIONS AND DATA ANALYSIS}

\subsection{Basic Measurements}

The 25 sources presented here are part of our sample of 40 $z \simeq 4.8$ luminous, unobscured, AGNs selected from the Sloan Digital Sky Survey (SDSS) and described in detail in T11. The sources were selected from the SDSS/DR6 database, requiring $4.65<z<4.92$ and $f_{\lambda}(1450 \AA)>6 \times 10^{-18} \mathrm{erg} \mathrm{s}^{-1} \AA^{-1}$, to ensure sufficient $H$-band brightness. Since the sources were optically selected, they are not biased with regards to SF properties. The redshift of 4.8 was chosen to allow the measurement of the $\mathrm{Mg}$ II $\lambda 2798$ emission line and the AGN continuum luminosity at rest-frame $3000 \AA$, using $H$-band spectroscopy. These allow a reliable measurement of $M_{\mathrm{BH}}$ and a normalized accretion rate, $L / L_{\text {Edd }}$ (McLure \& Dunlop 2004). Based on this sample, T11 showed that the highest luminosity AGNs at $z \simeq 4.8$ have, on average, lower $M_{\mathrm{BH}}$ and higher $L / L_{\mathrm{Edd}}$ than the highest luminosity AGNs in the corresponding samples of sources at $z \simeq 2-3.5$ from Shemmer et al. (2004) and Netzer et al. (2007a). Most of the $z \simeq 4.8$ SMBHs seem to be at the end of their first continuous growth phase that started at $z \gtrsim 10$ and are on their way to becoming the most massive $\left(>10^{10} M_{\odot}\right)$ black holes in the universe. An additional object, J1619+1238, was selected as part of the $z \simeq 4.8$ campaign described in T11, but the low quality of its $H$-band spectrum prohibited a reliable $M_{\mathrm{BH}}$ estimate, and thus it was not included in T11.

So far, 33 of the total $41 z \simeq 4.8$ sources have been observed with the SPIRE instrument (Griffin et al. 2010) on board Herschel, providing images at 250, 350, and $500 \mu \mathrm{m}$, corresponding to the rest-frame far-infrared (FIR) wavelength range of $43-86 \mu \mathrm{m}$. All SPIRE observations were made in the small-map mode, which is most suitable for point sources. The data reduction process starts with the level 0.5 product of the SPIRE pipeline. We then apply the standard tools, which are provided by the Herschel Science Centre (HSC) via the HIPE software (Ott 2010, version 7.3), and use the version 7.0 of calibration files. Since all of our sources appear as point sources in the images, we follow the guidelines of the HSC and apply a peak fitting method in order to measure the total flux. This is done by fitting a two-dimensional fixed-width Gaussian function to the image and taking the value at its peak to be the flux of the source.
In seven sources, the peak emission is shifted by $10^{\prime \prime}-30^{\prime \prime}$ from the optical location of the AGN. The low-resolution SPIRE images do not allow proper source separation in such cases, and deep observations at shorter wavelengths are needed to confirm these detections. A Spitzer campaign is underway to secure such observations, therefore we defer the analysis of these sources to a future publication. Of the remaining 26 sources, 5 were detected at above a $3 \sigma$ significance level in at least two SPIRE bands, and 20 were not detected at all SPIRE bands. One additional source (J1306+0236) was detected above a $3 \sigma$ level only in the $350 \mu \mathrm{m}$ band. In this Letter, we focus on two groups of sources either detected in at least two bands or undetected in all three bands. Therefore, we defer the analysis of this source to a future publication and hereafter refer only to the remaining 25 sources.

There are three sources of uncertainty related to the SPIRE images (see the SPIRE observers' manual ${ }^{5}$ ). The first uncertainty is in the fitted value and includes instrument and confusion noise. The integration time of each observation (either 222 or 296 seconds) is long enough to minimize the instrument noise and obtain an image that is dominated by the extragalactic confusion noise. The confusion noise is estimated to be 5.8, 6.3 , and $6.8 \mathrm{mJy}^{\text {beam }}{ }^{-1}$ at 250,350 , and $500 \mu \mathrm{m}$, respectively (Nguyen et al. 2010). The pixelization correction of the images introduces an uncertainty of about $2 \%-3 \%$ of the flux density. An additional uncertainty is associated with the calibration process and is about $7 \%$ of the flux density. These uncertainties are added in quadrature and listed together with the measured fluxes in Table 1.

\subsection{SED Fitting and Stacking Analysis}

The $350 \mu \mathrm{m}$ images of the five detected sources are shown in Figure 1, and their spectral energy distributions (SEDs) are shown in Figure 2. We also show the SDSS, $H$-, and $K$-band (where available) spectroscopy from $\mathrm{T} 11$, and the mean intrinsic mid-infrared (MIR) AGN SED from Mor \& Netzer (2012). The normalization of the MIR SED uses the known $L_{\mathrm{AGN}}$ and assumes a total covering factor by hot and warm dust of 0.5 . As discussed in Mor \& Netzer (2012), this value is close to the upper limit for such luminous AGNs. The AGN contribution to the emission at wavelengths longer than $\sim 30 \mu \mathrm{m}$ is small and its effect on the measurement of $L_{\mathrm{SF}}$ is negligible.

To measure $L_{\mathrm{SF}}$ we fitted the Herschel data points of each of the detected sources using a grid of templates that span

\footnotetext{
5 http://herschel.esac.esa.int/Docs/SPIRE/html/spire_om.html
} 


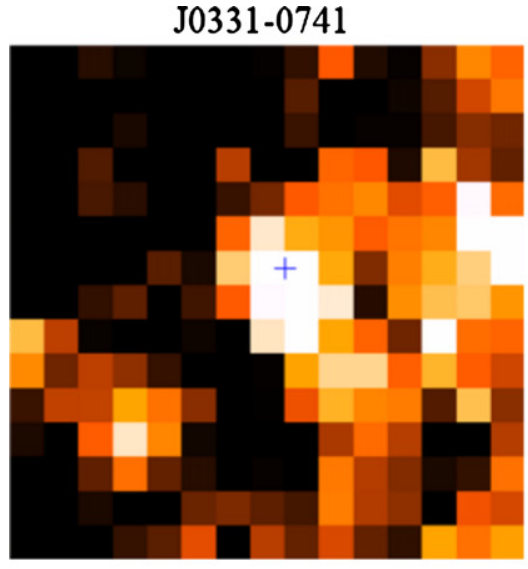

$\mathrm{J} 1619+1238$

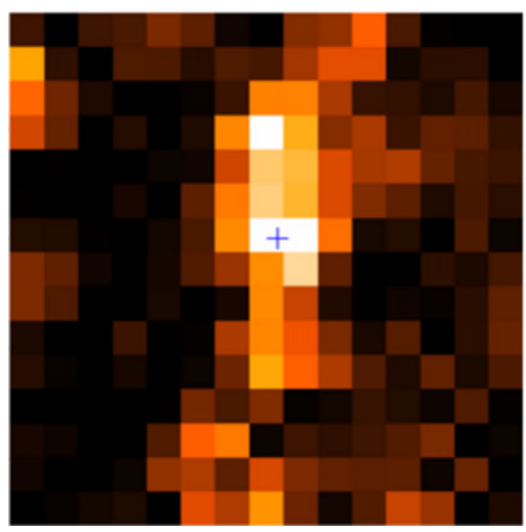

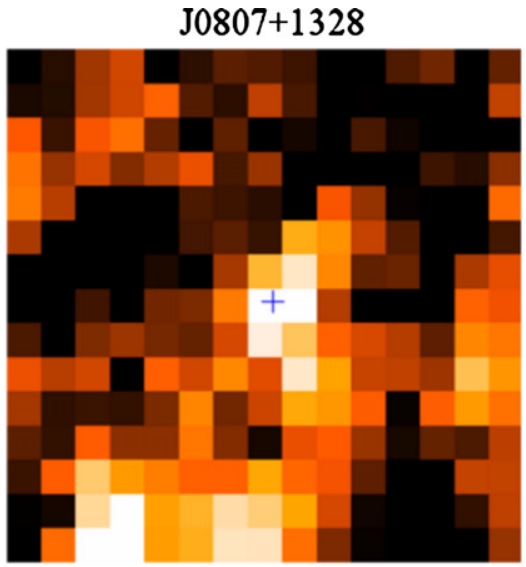

$\mathrm{J} 2225-0014$

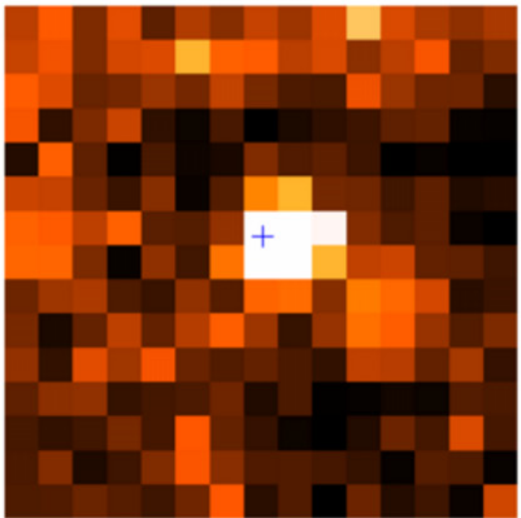

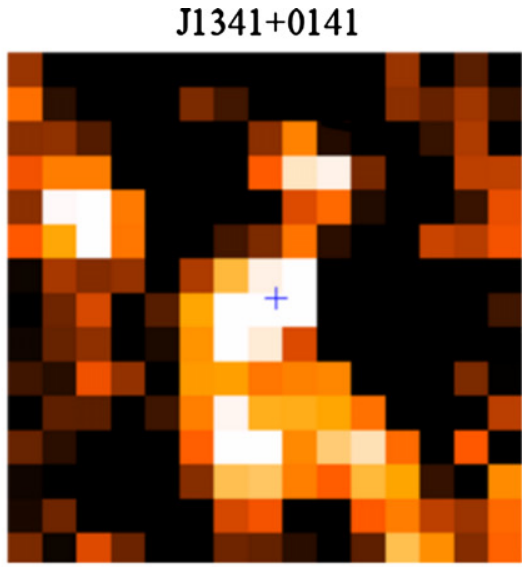

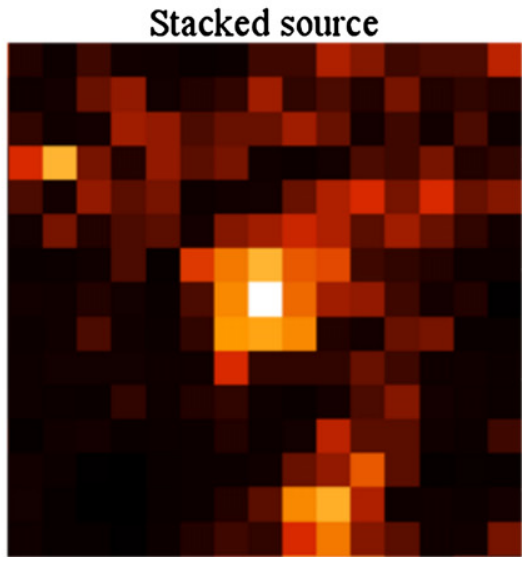

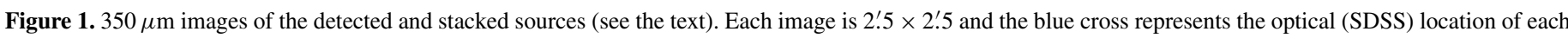
of the individually detected sources.

(A color version of this figure is available in the online journal.)

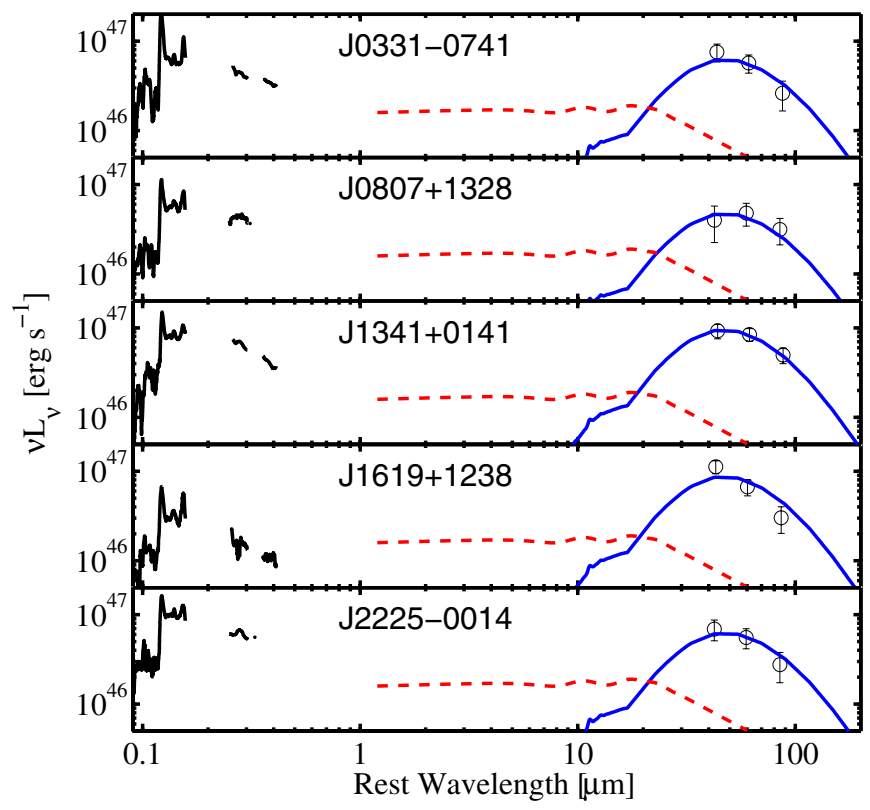

Figure 2. Spectral energy distributions (SEDs) of the five individually detected sources. The Herschel/SPIRE detections are shown as circles in each panel, and the best-fit luminous SF templates are shown as solid blue lines. The black lines at the short wavelength end represent SDSS spectra supplemented with $H$ - and $K$-band spectroscopy (where available) from Trakhtenbrot et al. (2011). Red dashed lines are mean intrinsic MIR AGN SEDs from Mor \& Netzer (2012) (see the text).

(A color version of this figure is available in the online journal.) a wide range of FIR luminosities and SFRs (Chary \& Elbaz 2001). The FIR luminosity is the only free parameter for this set of templates. $L_{\mathrm{SF}}$ is calculated by integrating the best-fit model between 8 and $1000 \mu \mathrm{m}$. Comparison with other types of models which are often used to estimate FIR luminosities (e.g., gray body) is beyond the scope of this Letter. The fitting procedure uses a standard $\chi^{2}$ minimization to determine the best-fit template. The uncertainty is calculated using the standard confidence levels for $\chi^{2}$ with two degrees of freedom.

As noted, 20 sources were not detected above $3 \sigma$ level at any of the bands. To get an average luminosity of these sources, we applied a stacking analysis to all 20 images in each band. We first cut each image to a small stamp symmetrically around the center of the pixel in which the optical location of the source lies. All stamps have an equal number of pixels and are approximately $1^{\prime} \times 1^{\prime}$ in size. A stacked image is constructed by assigning the images with weights according to their respective exposure times, and averaging the images pixel by pixel. Since the dominant source of uncertainty is the extragalactic confusion noise, the slightly different exposure times have a negligible effect. The stacking procedure revealed a statistically significant signal at all three bands (hereafter "stacked source") that represents the average flux of the individually undetected sources. We then measure the flux of the stacked source by fitting a two-dimensional Gaussian function as described above.

The averaging of the images assumes that all objects are located at the center. Any contribution from neighboring sources would be significantly reduced due to the fact that such 


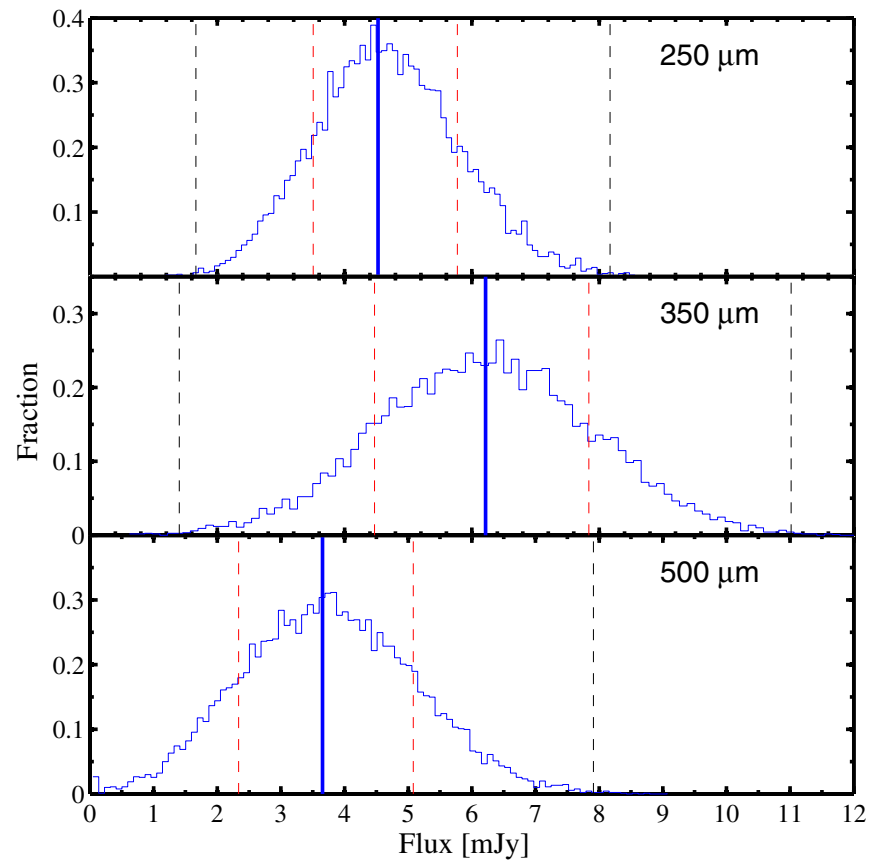

Figure 3. Probability distribution functions of the fluxes of the stacked sources (thin solid blue lines). The vertical solid lines mark the peaks (the most likely value) of the distributions. Inner and outer dashed lines represent the different percentiles corresponding to $1 \sigma$ and $3 \sigma$ uncertainties, respectively.

(A color version of this figure is available in the online journal.)

contributions are expected to be randomly distributed in the images. The average values can be biased if the (undetected) sources have very different fluxes. In such a case, a few sources that are just below the confusion noise limit might skew the result toward a higher average flux. To overcome this we used a bootstrap approach to estimate the true value of the flux of the stacked source and its uncertainty. Out of the 20 images we choose 10,000 random multisets of length 20. In each multiset, images may appear more than once. We then stacked each multiset of images and measured the flux of the emergent stacked source. The probability distribution functions (PDFs) of the bootstrap procedure in all three SPIRE bands are shown in Figure 3. The final value of the flux in each band is taken to be the maximum likelihood value of the corresponding distribution. Its uncertainty is estimated by measuring the 16th and 84th percentiles, which are assumed to represent the $1 \sigma$ error. The measured fluxes of the stacked source, are listed in Table 1. To measure the $L_{\mathrm{SF}}$ of the stacked source we follow the same fitting procedure as described above.

\section{RESULTS AND DISCUSSION}

Table 1 lists $L_{\mathrm{AGN}}$ (taken from T11), $L_{\mathrm{SF}}$, and SFRs for the detected and stacked sources. SFRs were calculated from $L_{\mathrm{SF}}$ assuming a Salpeter (1955) IMF and using $\mathrm{SFR}=1.73 \times$ $10^{-10} L_{\mathrm{SF}} / L_{\odot}$ (Kennicutt 1998). A top-heavy Kroupa IMF (Kroupa 2001) would result in SFRs that are lower by a factor of $\sim 1.6$.

We have collected from the literature FIR and sub-mm measurements of other luminous AGNs that imply $L_{\mathrm{SF}}>$ $5 \times 10^{12} L_{\odot}$. Eight sources at $z \sim 4$ have a single $850 \mu \mathrm{m}$ detection (Isaak et al. 2002). Four sources have been reported by Priddey et al. (2003). Two of these, at $z \sim 5$ and $z \sim 6$, have a single $850 \mu \mathrm{m}$ detection and two others, at similar redshifts, have both $850 \mu \mathrm{m}$ and $250 \mathrm{GHz}$ detections. Eight additional sources at $z \sim 5.8-6.2$ were detected at $250 \mathrm{GHz}$ (Wang et al. 2008, 2011). Finally, Leipski et al. (2010) reported multi-band measurements of two sources at $z \simeq 4.7$ and 6.4. We used the fluxes reported in these papers and applied our fitting method to obtain $L_{\mathrm{SF}}$. Fitting SF templates to one or two data points is problematic in two ways. First, a single data point fit does not allow the calculation of a meaningful confidence limit. Second, the $850 \mu \mathrm{m}$ and $250 \mathrm{GHz}$ bands translate to about $170 \mu \mathrm{m}$ restframe wavelength at the reported redshifts. This wavelength is far from the peak wavelength emission of cool dust. To estimate the range of possible $L_{\mathrm{SF}}$ for the sources with a single data point, we fitted the single-band measurement with a gray body with an emissivity index of $\beta=1.5$ and two temperatures, 40 and $60 \mathrm{~K}$. These temperatures represent the uncertainty in $L_{\mathrm{SF}}$ for these sources. $L_{\mathrm{AGN}}$ for these sources were calculated using the continuum flux density at $1450 \AA$ and the relation $\log \left(L_{\mathrm{AGN}} / 10^{46} \mathrm{erg} \mathrm{s}^{-1}\right)=0.94 \log \left(L_{1450} / 10^{46} \mathrm{erg} \mathrm{s}^{-1}\right)+0.53$, where $L_{1450}=\lambda L_{\lambda}(1450 \AA)$. This relation is based on several samples of high-z, high $L_{\mathrm{AGN}}$ sources (Shemmer et al. 2004; Netzer et al. 2007a; and T11).

Figure 4 summarizes the main results of this study on the $L_{\mathrm{SF}}$ versus $L_{\mathrm{AGN}}$ diagram. It shows a collection of several samples of low- and intermediate-luminosity AGN-dominated sources from Netzer (2009), the high-luminosity, high-redshift sources collected from the literature, and our $z \simeq 4.8$ sources. The AGN-dominated correlation line and a 1:1 line are also shown.

The two main results of our work are best explained by considering the data shown in the inset of Figure 4. First, the SFRs of the five individually detected sources are comparable to the most FIR luminous objects known, including SMGs. Thus, the most intense SF phase in AGN hosts is not necessarily associated with an obscured SMBH phase. Second, the detected and stacked sources occupy two distinct locations on the $L_{\mathrm{AGN}}-L_{\mathrm{SF}}$ plane. The mean SFR of the detected sources is higher than the SFR of the stacked source by about a factor of five. This result is consistent with the findings of Wang et al. (2011) for a sample of $z \sim 6$ quasars. All the sources in our sample have similar optical-UV spectra, span a narrow range of $M_{\mathrm{BH}}$ values, similar high values of $L_{\mathrm{AGN}}$, and almost identical redshifts (see Table 1). Thus, the AGN properties do not explain the different SF properties.

We consider three scenarios to explain the observed different SFRs in the two groups.

1. The different locations represent different evolutionary routes. The detected sources are in major mergers with extremely high SFRs and the undetected ones go through a calmer secular evolution with lower SFRs. The similar AGN properties and different SFRs of the sources in both groups imply different relative growth rates of the SMBH and stellar mass in each group. Assuming that the ratios of growth rates remain the same for sometime (before or after $z=4.8$ ), this will lead to different $M_{*} / M_{\mathrm{BH}}$ at the end of their evolution. Since all the sources are probably on their way to becoming the most massive black holes in the universe (T11), this scenario is not supported by observations in the local universe.

2. All the sources are in merging systems where an initial burst of SF is followed by accretion onto one or two SMBHs. The different SFRs may be explained by different conditions in the mergers (e.g., galaxy size and gas content). If this is correct, then the SFRs of the sources should have been more evenly distributed across the SFR range. Furthermore, 


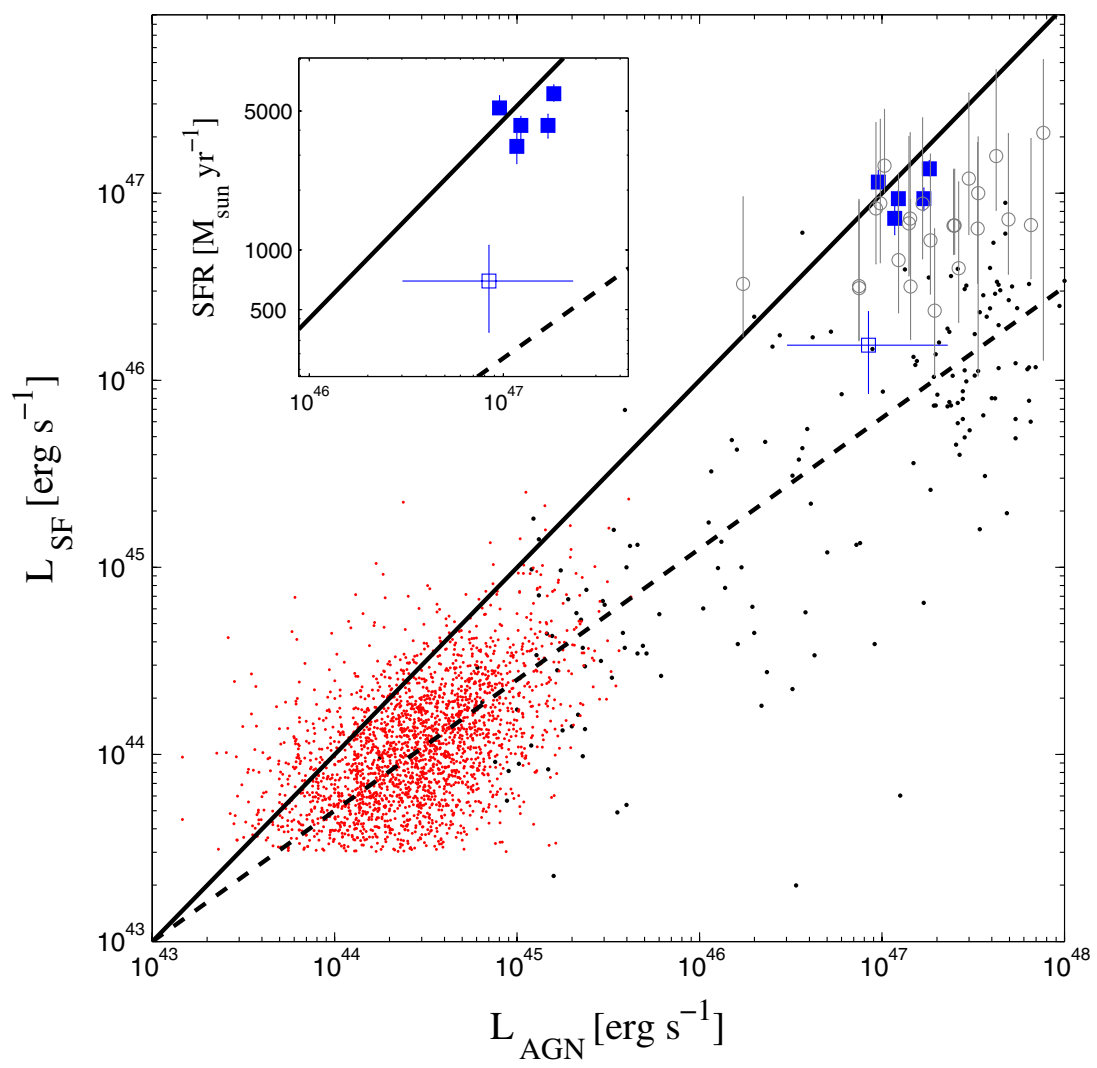

Figure 4. AGN luminosity, $L_{\mathrm{AGN}}$, vs. star formation luminosity, $L_{\mathrm{SF}}$, for several samples. The five $z \simeq 4.8$ sources detected using the Herschel/SPIRE are shown as blue squares. The value of the stacked source is represented by the blue empty square. The horizontal error bar represents the range of $L_{\mathrm{AGN}}$ for the group of undetected sources, and the vertical error bar is set by the $3 \sigma$ confidence level of the PDFs in Figure 3. Dots represent data from several samples of type-I AGN at low and high redshifts (black dots) and type-II AGNs (smaller red dots), see the text. The main correlation for AGN dominated sources is shown as a dashed line and can be expressed as $L_{\mathrm{SF}} \simeq 10^{43}\left(L_{\mathrm{AGN}} /\left(10^{43} \mathrm{erg} \mathrm{s}^{-1}\right)\right)^{0.7}$. A 1:1 ratio is shown as a solid line for comparison. Open gray circles represent observations collected from the literature. Their $L_{\mathrm{SF}}$ determination is based, in most cases, on a single sub-mm point (see the text). The inset shows $L_{\mathrm{AGN}}$ vs. SFR for the new $z \simeq 4.8$ data.

(A color version of this figure is available in the online journal.)

in this case the gas supply to the SMBH is only loosely connected with the SF in the host.

3. All the sources reside in merging systems of similar type and the different SFRs are due to different stages of the merger process. The process can continue in two different ways. The first is that both SFR and $L / L_{\text {Edd }}$ keep their observed values for a certain time, i.e., $L_{\mathrm{SF}}$ remains roughly constant while $L_{\mathrm{AGN}}$ increases with time. In terms of the $L_{\mathrm{AGN}}-L_{\mathrm{SF}}$ diagram, it means that sources start their fastest SF growth phase at some point in the diagram and travel horizontally, similar to the suggestion of Shao et al. (2010). However, if our five individually detected sources were to follow this path, they would reach the AGN-dominated correlation line, at lower redshift, with $L_{\mathrm{AGN}} \geqslant 10^{49} \mathrm{erg} \mathrm{s}^{-1}$. Such luminous AGNs are extremely rare at $z \sim 2$ and have never been observed at $z>3$, but in our sample they represent $25 \%$ of the population. The second possibility is that the five detected sources are at the peak of the SF phase and the other 20 toward its end. Given the similar redshift of the sources, the SF must have been reduced significantly over a short period of time, while their $L_{\mathrm{AGN}}$ remained at its peak. The redshift range of the sample $(4.65<z<4.92)$ implies a timescale of $\sim 100 \mathrm{Myr}$ for this process. In terms of the $L_{\mathrm{AGN}}-L_{\mathrm{SF}}$ plane, this means that the 20 sources with lower $L_{\mathrm{SF}}$ had higher SFR in the past but traveled vertically down toward the AGN-dominated correlation line. A possible way to rapidly quench SF is by AGN feedback. In this scenario, which we consider more probable, feedback reduced SF in the hosts of the 20 lower- $L_{\mathrm{SF}}$ objects but is not yet operating efficiently in the other five. If this scenario is true, then the AGN activity should be significantly reduced shortly after the quenching of the SF. This is consistent with the estimate of the AGN duty cycle by $\mathrm{T} 11$.

To conclude, our optically selected, flux-limited sample, which consists of sources that span a narrow range of AGN properties, provides an excellent test-bed of various SMBH and galaxy evolution scenarios at $z \simeq 4.8$. The extremely high SFRs found in five sources provide strong evidence for a merger process in these systems. The clear separation into two groups with SFRs that differ by a large factor provides, perhaps, indications for AGN feedback.

This work is based on observations made with Herschel, an European Space Agency Cornerstone Mission with significant participation by NASA. Support for this work was provided by NASA through an award issued by JPL/Caltech. We are grateful to A. Sternberg and D. Lutz for useful discussions and comments. An anonymous referee made useful comments that helped improve this Letter. We thank the DFG for support via German Israeli Cooperation grant STE1869/1-1.GE625/15-1. Funding for this work has also been provided by the Israel Science Foundation grant 364/07. P.L. acknowledges funding support from the Fondecyt project number 1080603. 


\section{REFERENCES}

Chary, R., \& Elbaz, D. 2001, ApJ, 556, 562

Di Matteo, T., Colberg, J., Springel, V., Hernquist, L., \& Sijacki, D. 2008, ApJ, 676, 33

Di Matteo, T., Springel, V., \& Hernquist, L. 2005, Nature, 433, 604

Griffin, M. J., Abergel, A., Abreu, A., et al. 2010, A\&A, 518, L3

Guyon, O., Sanders, D. B., \& Stockton, A. 2006, ApJS, 166, 89

Hatziminaoglou, E., Omont, A., Stevens, J. A., et al. 2010, A\&A, 518, L33

Hopkins, P. F., Hernquist, L., Cox, T. J., et al. 2006, ApJS, 163, 1

Isaak, K. G., Priddey, R. S., McMahon, R. G., et al. 2002, MNRAS, 329, 149

Kennicutt, R. C. 1998, ARA\&A, 36, 189

Kroupa, P. 2001, MNRAS, 322, 231

Leipski, C., Meisenheimer, K., Klaas, U., et al. 2010, A\&A, 518, L34

Lutz, D., Sturm, E., Tacconi, L. J., et al. 2008, ApJ, 684, 853

McLure, R. J., \& Dunlop, J. S. 2004, MNRAS, 352, 1390

Mor, R., \& Netzer, H. 2012, MNRAS, 420, 526

Netzer, H. 2009, MNRAS, 399, 1907

Netzer, H., Lira, P., Trakhtenbrot, B., Shemmer, O., \& Cury, I. 2007a, ApJ, 671, 1256

Netzer, H., Lutz, D., Schweitzer, M., et al. 2007b, ApJ, 666, 806
Nguyen, H. T., Schulzw, E., Levenson, L., et al. 2010, A\&A, 518, L5

Ott, S. 2010, in ASP Conf. Ser.434, Astronomical Data Analysis Software and Systems XIX, ed. Y. Mizumoto, K.-I. Morita, \& M. Ohishi (San Francisco, CA: ASP), 139

Priddey, R. S., Isaak, K. G., McMahon, R. G., Robson, E. I., \& Pearson, C. P. 2003, MNRAS, 344, L74

Rodighiero, G., Daddi, E., Baronchelli, I., et al. 2011, ApJ, 739, L40

Salpeter, E. E. 1955, ApJ, 121, 161

Shao, L., Lutz, D., Nordon, R., et al. 2010, A\&A, 518, L26

Shemmer, O., Netzer, H., Maiolino, R., et al. 2004, ApJ, 614, 547

Sijacki, D., Springel, V., Di Matteo, T., \& Hernquist, L. 2007, MNRAS, 380, 877

Sijacki, D., Springel, V., \& Haehnelt, M. G. 2011, MNRAS, 414, 3656

Springel, V., Di Matteo, T., \& Hernquist, L. 2005, MNRAS, 361, 776

Trakhtenbrot, B., Netzer, H., Lira, P., \& Shemmer, O. 2011, ApJ, 730, 7 (T11)

Valiante, R., Schneider, R., Salvadori, S., \& Bianchi, S. 2011, MNRAS, 416, 1916

Wang, R., Carilli, C. L., Wagg, J., et al. 2008, ApJ, 687, 848

Wang, R., Wagg, J., Carilli, C. L., et al. 2011, AJ, 142, 101

Wu, J., Vanden Bout, P. A., Evans, N. J., \& Dunham, M. M. 2009, ApJ, 707, 988 